\title{
FACTORIZATION IN BANACH ALGEBRAS AND THE GENERAL STRICT TOPOLOGY
}

\author{
BY \\ F. DENNIS SENTILLES AND DONALD CURTIS TAYLOR
}

1. Introduction. Let $C(S)$ be the usual Banach space of bounded complex valued continuous functions on a locally compact Hausdorff space $S$ and let $C_{0}(S)$ be the subspace of all functions in $C(S)$ that vanish at infinity. In $1958 \mathrm{R}$. C. Buck [1] published a study of the linear space $C(S)$ under the $\beta$ or strict topology; that is, the weak topology generated by the Banach algebra of bounded linear operators on $C(S)$

$$
T_{\phi}(f)=\phi f \quad(f \in C(S)),
$$

where $\phi$ ranges over $C_{0}(S)$, or, equivalently, the topology generated by the collection of seminorms $f \rightarrow\|\phi f\|_{\infty}$ for $\phi \in C_{0}(S)$. It was shown that $C(S)_{\beta}$ is a complete locally convex topological vector space whose bounded sets coincide with the (supremum) norm bounded sets and whose strong dual is the Banach space of bounded regular Borel measures on $S$ under the variation norm; that is, the dual of $C(S)_{\beta}$ is identical to the dual of $C_{0}(S)=C_{0}(S) \cdot C(S)$. Subsequently, Conway [4] characterized the $\beta$-equicontinuous subsets of the dual and showed that when $S$ is paracompact the strict topology is the Mackey topology on $C(S)$. For the most part, both Buck and Conway utilize a constructive process in the proofs of their theorems (for example, see [1, Theorem 2, p. 99] and [4, Theorem 2.2, p. 476]) and, apparently, the essential ingredient of their arguments is the existence of an approximate identity in $C_{0}(S)$.

The purpose of this paper is twofold. First, we will establish certain factorization theorems in Banach algebras with approximate identity (see $\$ 2$ ) and then, by virtue of these factorization theorems, we wish to suggest that the proper place to study the strict topology is in the following general setting (see $\S \S 3$ and 4 ). Let $B$ be a Banach algebra with approximate identity and let $X$ be a left Banach $B$-module. The strict topology on $X$ induced by $B$ is the locally convex topology generated by the seminorms $x \rightarrow\|T(x)\|$ for each $T \in B$. In support of our latter claim, we obtain (with the exception that the strict topology on $X$ is the Mackey topology) with surprising ease all of the above results, or applicable conditions equivalent to their validity. Finally, we apply this work to several interesting examples (see $§ 5)$.

Presented to the Society, April 20, 1968; received by the editors March 7, 1968 and, in revised form, November 8, 1968. 
The authors wish to thank the referee for calling our attention to the work of Rieffel [11], and for his other comments and recommendations.

2. Factorization theorems for Banach modules. In this section we give certain basic definitions and state several results concerning factorization in Banach modules over Banach algebras.

TheOREM 2.1. Suppose $B$ is a Banach algebra with bounded left approximate identity $\left\{E_{\lambda}\right\}$ of norm $C$, and suppose $X$ is an essential left Banach B-module (see $[11, p .453])$. If $Z$ is a uniformly bounded subset of $X$, then the following statements are equivalent :

(1) There exists a $T \in B$ and a uniformly bounded subset $W$ of $X$ such that $Z=T(W)$.

(2) $\left\|E_{\lambda}(z)-z\right\| \rightarrow 0$ uniformly on $Z$.

(3) For each $\delta>0$ there exists $a T \in B,\|T\| \leqq C$, and a uniformly bounded subset $W$ of $X$ such that the following conditions are satisfied: The map $w \rightarrow T(w)$ is a homeomorphism of $W$ onto $Z$, and $\|T(w)-w\|<\delta$ for each $w \in W$.

Proof. It is clear that (1) implies (2), and (3) implies (1). Therefore, to complete the proof we need to show that (2) implies (3). Now assume (2) holds and $0<\delta<1$. Furthermore, we may assume, without loss of generality, that $Z$ is uniformly bounded by 1 . Let $B_{1}$ be the Banach algebra obtained by formally adjoining the unit $I$ to $B$; that is, $B_{1}=\{\alpha I+T: \alpha$ complex, $T \in B\}$ under the norm $\|\alpha I+T\|$ $=|\alpha|+\|T\|$. It is easy to see that if we view $I$ as the identity operator on $X$, then $X$ is an essential left Banach $B_{1}$-module. Let $0<\nu<1 / 4 C$. Since $\left\|E_{\lambda}(z)-z\right\| \rightarrow 0$ unformly on $Z$, we may choose an element $E_{1}$ in $B,\left\|E_{1}\right\| \leqq C$, such that $\left\|E_{1}(z)-z\right\|$ $<\delta / 4, z \in Z$. Again, since $\left\|E_{\lambda}(z)-z\right\| \rightarrow 0$ uniformly on $Z$, we may define by induction a sequence of elements $E_{1}, E_{2}, E_{3}, \ldots$ in $B$ so that for each positive integer $n$ the following conditions are satisfied:

$$
\begin{gathered}
\left\|E_{n+1}\right\| \leqq C, \quad\left\|E_{n+1}(z)-z\right\|<\delta / 8^{n+1} \quad(z \in Z), \quad \text { and } \\
\left\|E_{k}-E_{n+1} E_{k}\right\|<\delta / 32^{n+1} \quad(k=1,2, \ldots, n) .
\end{gathered}
$$

Now set

$$
T_{n}=\sum_{k=1}^{n} \nu(1-\nu)^{k-1} E_{k}+(1-\nu)^{n} I
$$

It is easy to see that $T_{1}^{-1}$ exists and that

$$
\left\|T_{1}^{-1}\right\| \leqq 4, \quad\left\|T_{1}^{-1}(z)-z\right\|<\delta / 4 \quad(z \in Z) .
$$

Now assume that for the positive integer $n$ that $T_{n}^{-1}$ exists and $\left\|T_{n}^{-1}\right\| \leqq 4^{n}$. We shall prove that $T_{n+1}^{-1}$ exists and $\left\|T_{n+1}^{-1}\right\| \leqq 4^{n+1}$. Set $G=(1-\nu)^{n}\left[(1-\nu) I+\nu E_{n+1}\right]$. It is clear that $G^{-1}$ exists, $\left\|G^{-1}\right\| \leqq 2(1-\nu)^{-n}$, and $(1-\nu)^{n} G^{-1}=I+\nu(1-\nu)^{n} G^{-1}\left(I-E_{n+1}\right)$. 
Thus

$$
\begin{aligned}
T_{n+1} & =(1-\nu)^{-n} G\left[T_{n}+\nu(1-\nu)^{n} G^{-1} \sum_{k=1}^{n} \nu(1-\nu)^{k-1}\left(E_{k}-E_{n+1} E_{k}\right)\right] \\
& =(1-\nu)^{-n} G T_{n} H,
\end{aligned}
$$

where $H=I+\nu(1-\nu)^{n} T_{n}^{-1} G^{-1} \sum_{k=1}^{n} \nu(1-\nu)^{k-1}\left(E_{k}-E_{n+1} E_{k}\right)$. Due to the fact that $\left\|T_{n}^{-1}\right\| \leqq 4^{n}$, we have by (2.1) that the norm $\|I-H\| \leqq \nu \delta / 2^{3 n+2}$. Thus $H^{-1}$ exists and $H^{-1}=I+H^{\prime}$, where $H^{\prime}=\sum_{k=1}^{\infty}(I-H)^{k}$. This implies that $T_{n+1}^{-1}$ exists and the norm $\left\|T_{n+1}^{-1}\right\| \leqq 4^{n+1}$. Furthermore, it follows from (2.4) that

$$
\begin{aligned}
T_{n+1}^{-1} & =H^{-1} T_{n}^{-1}\left[(1-\nu)^{n} G^{-1}\right] \\
& =\left(I+H^{\prime}\right)\left(T_{n}^{-1}\right)\left(I+\nu(1-\nu) G^{-1}\left(I-E_{n+1}\right)\right) \\
& =T_{n}^{-1}+R\left(I-E_{n+1}\right)+S,
\end{aligned}
$$

where $R=\nu(1-\nu)^{n}\left(T_{n}^{-1}+H^{\prime} T_{n}^{-1}\right) G^{-1}$ and $S=H^{\prime} T_{n}^{-1}$. Since $\|R\| \leqq 4^{n}$ and $\|S\| \leqq \delta / 2^{n+2}$, we have by virtue of $(2.1)$

$$
\left\|T_{n+1}^{-1}(z)-T_{n}^{-1}(z)\right\|<\delta / 2^{n+1} \quad(z \in Z) .
$$

The elements $\left\{T_{n}^{-1}(z)\right\}$ form a Cauchy sequence in $X$ for each $z \in Z$. Therefore set

$$
W=\left\{w=\lim T_{n}^{-1} z: z \in Z\right\} \quad \text { and } \quad T=\sum_{k=1}^{\infty} \nu(1-\nu)^{k-1} E_{k} .
$$

Since $T_{n}^{-1} z \rightarrow w$ uniformly on $Z$, we see that the map $w \rightarrow T(w)$ is a homeomorphism of $W$ onto $Z$. To see that $\|T(w)-w\|<\delta$, for each $w \in W$, we observe that the inequalities

$$
\begin{aligned}
\|T(w)-w\| \leqq & \left\|T(w)-T_{1}^{-1}(T(w))\right\|+\sum_{j=1}^{n-1}\left\|T_{j+1}^{-1}(T(w))-T_{j}^{-1}(T(w))\right\| \\
& +\left\|T_{n}^{-1}(T(w))-w\right\|<\delta
\end{aligned}
$$

hold for each element $w$ in $W$ for the appropriate choice of $n$. Hence our proof is complete.

We remark that if $X$ is a right module and $\left\{E_{\lambda}\right\}$ is a right approximate identity for $B$, then a right-hand version of Theorem 2.1 holds.

Theorem 2.1 is, of course, a generalization of Cohen's factorization theorem [2], and other forms of Theorem 2.1 have already been proven. For example, Rieffel [12] showed, by means of a simple device, that if $Z$ is a sequence of elements in $X$ that converges to zero, then Theorem 2.1 is a corollary of a result found by Hewitt [7], Curtis and Figá-Talamanca [5], and Gulick, Liu, and van Rooij [6]. More recently, Collins and Summers [3] introduced an auxiliary left Banach $B$-module and then applied Hewitt's factorization theorem to improve the method of proof of our Theorem 2.1. Our contribution is that we give a simple method for determining whether or not certain "large" sets have common factors, and the 
value of this will become apparent when we begin our study of the generalized strict topology.

THEOREM 2.2. Let $X$ be a Banach space, $B$ a Banach algebra of linear operators on $X$ under the usual operator norm, and let $X_{e}$ denote the closed linear subspace of $X$ spanned by $B X$. If each operator $T \in B$ is compact and $C \geqq 1$, then the following statements are equivalent:

(1) If $Z$ is a finite subset of $X_{e}$ and $\delta>0$, then there exists an operator $T$ in $B$, $\|T\| \leqq C$, and a finite subset $W$ of $X$ such that $Z=T(W)$ and $\|T(w)-w\|<\delta(w \in W)$.

(2) $B$ has a left approximate identity of norm $\leqq C$.

Proof. It follows directly from Theorem 2.1 that (2) implies (1). Therefore, to complete the proof, we need to show that (1) implies (2). Suppose (1) holds and that $F$ is the family of all finite subsets of $X_{e}$. Let $\Lambda$ be the directed set defined by

$$
\Lambda=\{(Z ; n): Z \in F, n \text { a positive integer }\}
$$

under the usual partial ordering; that is, $\left(Z_{1}, n_{1}\right) \leqq\left(Z_{2}, n_{2}\right)$ if, and only if, $Z_{1} \subset Z_{2}$ and $n_{1} \leqq n_{2}$. For each $\lambda=(Z, n) \in \Lambda$ we may choose, by virtue of (1), an operator $E_{\lambda}$ in $B,\left\|E_{\lambda}\right\| \leqq C$, and a finite subset $W_{\lambda}$ of $X$ such that

(a) $Z=E_{\lambda} W_{\lambda}$.

(b) $\left\|E_{\lambda}(w)-w\right\|<1 / n\left(w \in W_{\lambda}\right)$.

Now, for arbitrary $T \in B$ and $\varepsilon>0$, there exists, by the compactness of $T$, a finite subset $Y$ of the unit ball of $X$ such that

$$
\left\|E_{\lambda} T-T\right\|<\sup \left\{\left\|E_{\lambda}(T(y))-T(y)\right\|: y \in Y\right\}+\varepsilon / 2
$$

for each $\lambda$. Choose $\lambda_{0}=(T(Y), n)$, where $C / n<\varepsilon / 2$. Then for $\lambda \geqq \lambda_{0}$ we have, by virtue of (2.6)

$$
\begin{aligned}
\left\|E_{\lambda} T-T\right\| & <\sup \left\{\left\|E_{\lambda}\left(E_{\lambda}(w)\right)-E_{\lambda}(w)\right\|: w \in W_{\lambda}\right\}+\varepsilon / 2 \\
& \leqq C / n+\varepsilon / 2<\varepsilon .
\end{aligned}
$$

In other words, $\lim E_{\lambda} T=T$ for every $T \in B$. Hence (1) implies (2).

THEOREM 2.3. Let $B$ be a Banach algebra and let $C \geqq 1$. Then the following statements are equivalent:

(1) If $Z$ is a finite subset of $B$ and $\delta>0$, then there exists an element $x$ in $B$, $\|x\| \leqq C$, and a subset $W$ of $B$ such that $Z=x W$ and $\|x w-w\|<\delta(w \in W)$.

(2) $B$ has a left approximate identity of norm $\leqq C$.

Proof. It follows directly from Theorem 2.1, that (2) implies (1). To see that (1) implies (2), we refer the reader to [16].

THEOREM 2.4. Let $B$ be a Banach algebra with approximate identity $\left\{E_{\lambda}\right\}$, and let $X$ be a left Banach $B$-module. Then the following statements are equivalent:

(1) There exists an element $T$ in $B$ such that the map $x \rightarrow T(x)$ is an isomorphism of $X$ onto $X$. 
(2) There exists an element $T$ in $B$ such that the map $x \rightarrow T(x)$ is an isomorphism of $X$ onto a closed subspace of $X$.

(3) $\left\|E_{\lambda}(x)-x\right\| \rightarrow 0$ uniformly on the unit ball of $X$.

Proof. It is clear that (1) implies (2). Now assume (2) holds and $T$ is an element in $B$ such that the map $x \rightarrow T(x)$ is an isomorphism of $X$ onto a closed subspace of $X$. By the open mapping theorem there exists a constant $N$ such that $\|x\|$ $\leqq N\|T(x)\|$ for each $x \in X$ and this implies that

$$
\left\|E_{\lambda}(x)-x\right\| \leqq N\left\|T\left(E_{\lambda}(x)-x\right)\right\| \leqq N\left\|T E_{\lambda}-T\right\|\|x\| .
$$

In other words, $E_{\lambda}(x) \rightarrow x$ uniformly on the unit ball of $X$. Hence (2) implies (3).

To complete the proof, we need to show (3) implies (1). Let $C$ be a constant such that $\left\|E_{\lambda}\right\| \leqq C$ for each $\lambda$ and let $\delta=1 / 2 C$. According to Theorem 2.1 there exists an element $T$ in $B,\|T\| \leqq C$, and uniformly bounded subset $W$ of $X$ such that

(a) $\{x \in X:\|x\| \leqq 1\}=T(W)$.

(b) $\|T(w)-w\|<1 / 2 C(w \in W)$.

Suppose that the map $x \rightarrow T(x)$ is not one-to-one. Then there exists an $x \in X$, $\|x\|=1$, such that $T(x)=0$. Thus there exists a $w \in W$ such that

$$
1=\|x\|=\|x-T(x)\|=\|T(w)-T(T(w))\| \leqq C\|w-T(w)\|<1 / 2 .
$$

Hence (3) implies (1).

3. The general strict topology. Throughout the remainder of this paper let $B$ be a Banach algebra with approximate identity $\left\{E_{\lambda}\right\}$ of norm one, let $X$ be a left Banach $B$-module, and let $X_{e}$ denote the essential part of $X$ (see [11, p. 454]). We shall also assume that if $x \in X, x \neq \theta$, then there is a $T \in B$ such that $T x \neq \theta$. Under these hypotheses, the $\beta$ or strict topology induced on $X$ by $B$ by way of the seminorms $x \rightarrow\|T x\|, T \in B$, in the sense of $[13$, p. 15], is a locally convex Hausdorff topology on $X$. We will write $X_{\beta}$ to denote the linear space $X$ with the strict topology. In this section we study the space $X_{\beta}$ and in the next section its strong dual.

At this point it is convenient to relate $X$ to $\operatorname{Hom}_{B}\left(B, X_{e}\right)$, the linear space of all continuous operators $P$ on $B$ into $X_{e}$ such that $P(S T)=S P(T)$ for all $S, T \in B$. (See [11] and note that by [11, Corollary 3.8], $\operatorname{Hom}_{B}\left(B, X_{e}\right) \cong \operatorname{Hom}_{B}(B, X)$.) For $x \in X$ and $T \in B$ let $P_{x}(T)=T x$. Then $P_{x}(S T)=S T x=S P_{x}(T)$ and $P_{x}$ is a continuous linear operator that maps $B$ into $X_{e}$. Hence $P_{x} \in \operatorname{Hom}_{B}\left(B, X_{e}\right)$. The mapping $x \rightarrow P_{x}$ is an isomorphism of $X$ into $\operatorname{Hom}_{B}\left(B, X_{e}\right)$ and becomes an isometry if we renorm the space $X$ according to the formula

$$
\|x\|^{\prime}=\sup \{\|T x\|: T \in B,\|T\| \leqq 1\} .
$$

Hence $\|x\|^{\prime}$ is the operator norm of $P_{x}$ on $B$. We will denote the given norm topology on $X$ by $\sigma$ and the topology defined by $\|\cdot\|^{\prime}$ will be denoted by $\sigma^{\prime}$. Clearly $\sigma$ is finer than $\sigma^{\prime}$; their equivalence is considered in Theorem 4.6. All further notation and terminology is, unless otherwise specified, that of [13]. 
THEOREM 3.1. The collection of all sets $V_{T}=\{x:\|T x\| \leqq 1\}$ for $T \in B$ is a base for the neighborhood system at 0 in the strict topology.

Proof. By [13, p. 15] a base for the neighborhood system at 0 consists of sets of the form $\bigcap_{i=1}^{m}\left\{x:\left\|T_{i} x\right\| \leqq \varepsilon\right\}$ for a given $\varepsilon>0$ and $T_{1}, \ldots, T_{m} \in B$. By the righthand version of Theorem 2.3 (1), there is an $R \in B$ and such that $T_{i}=S_{i} R$ for some choice of $S_{1}, \ldots, S_{m} \in B$. Let $b=\max \left\{\left\|S_{i}\right\| / \varepsilon: i=1,2, \ldots, n\right\}$ and let $T=b R$. Then $\|T x\| \leqq 1$ implies $\left\|T_{i} x\right\|=\left\|S_{i} R x\right\| \leqq(1 / b)\left\|S_{i}\right\|\|b R x\| \leqq \varepsilon$.

The following result, along with Theorem 3.3, is an analogue of the result obtained by Buck [1, Theorem 1] for $C(S)_{\beta}$.

THEOREM 3.2. The following are equivalent:

(1) $E_{\lambda} x \rightarrow x$ uniformly on the unit ball in $X$.

(2) The $\beta$ and $\sigma$ topologies are equivalent topologies on $X$.

(3) $X_{\beta}$ is a Fréchet space.

(4) $X_{\beta}$ is barrelled.

Proof. By virtue of Theorem 2.4 and the open mapping theorem we see that (1) implies (2). If (2) holds, (3) is clear. Since any Fréchet space is barrelled and the $\sigma^{\prime}$ topology is finer than the strict topology, we see that (3) implies (4). Finally by (4), the identity map $I: X_{\sigma} \rightarrow X_{\beta}$ is 1-1 continuous linear from a Banach space onto a barrelled space and by [13, p. 116], $\beta=\sigma$, and (2) holds. But then (2) implies (1), completing the proof.

In general then, the strict topology is lacking in certain desirable properties. It is the purpose of the remainder of this paper to show that certain equally desirable properties are maintained.

Before considering the question of the completeness of $X_{\beta}$ we introduce an analogue of the compact-open topology on $C(S)$ and obtain a generalization of Buck's result.

Let $\kappa$ denote the locally convex topology induced on $X$ by the seminorms $x \rightarrow\left\|E_{\lambda} x\right\|$ in the sense of $\left[13\right.$, p. 15] where $\left\{E_{\lambda}\right\}$ is the approximate identity in $B$.

THEOREM 3.3.

(1) $\kappa$ is a Hausdorff topology and $\beta$ is finer than $\kappa$.

(2) $\kappa$ and $\beta$ agree on $\sigma^{\prime}$ bounded sets.

(3) The strict closure of $\bigcup_{\lambda} E_{\lambda} X$ is $X$.

(4) $A$ sequence $\left\{x_{n}\right\} \subset X$ is $\beta$ convergent if and only if it is $\sigma^{\prime}$ bounded and $\kappa$ convergent.

Proof of (1). If $E_{\lambda} x=0$ for all $\lambda$, then for any $T \in B, T x=0$ and we have $x=0$. Clearly $\beta$ is finer than $\kappa$.

Proof of (2). Let $\left\{x_{\gamma}\right\}$ be a $\sigma^{\prime}$ bounded and $\kappa$ convergent net to $x \in X$. Let $b=\sup \left\{\|x\|^{\prime},\left\|x_{\gamma}\right\|^{\prime}\right\}$. Let $\varepsilon>0$ and let $T \in B$.

Choose $\lambda$ such that $\left\|T E_{\lambda}-T\right\|<\varepsilon / 3 b$ and $\gamma_{0}$ such that $\gamma \geqq \gamma_{0}$ implies $\left\|T E_{\lambda}\left(x_{\gamma}-x\right)\right\|$ $<\varepsilon / 3$. 
Then for $\gamma \geqq \gamma_{0}$

$$
\left\|T x_{y}-T x\right\| \leqq\left\|T x_{y}-T E_{\lambda} x_{\gamma}\right\|+\left\|T E_{\lambda} x_{\gamma}-T E_{\lambda} x\right\|+\left\|T E_{\lambda} x-T x\right\|<\varepsilon .
$$

Hence $\left\{x_{\gamma}\right\}$ is strictly convergent to $x$.

Proof of (3). If $x \in X$ and $T \in B$, then $T x=\lim T E_{\lambda} x$. Hence (3) holds.

Proof of (4). If $\left\{x_{n}\right\}$ is $\sigma^{\prime}$ bounded and $\kappa$ convergent to $x$, then by (2) $\kappa$ and $\beta$ agree on the $\sigma^{\prime}$ bounded set $\left\{x, x_{n}\right\}$ and consequently $\left\{x_{n}\right\}$ is $\beta$ convergent to $x$.

Conversely, if $\left\{x_{n}\right\}$ is $\beta$ convergent to $x$, then $\left\{x_{n}\right\}$ is $\kappa$ convergent to $x$ and furthermore the operators $\left\{P_{x_{n}}\right\}$ being pointwise convergent and hence pointwise bounded must be uniformly bounded in the operator norm on $B$. This completes the proof since $\left\|x_{n}\right\|^{\prime}=\left\|P_{x_{n}}\right\|$.

Corollary 3.4. $X_{e}$ is strictly dense in $X$.

We now consider the question of completeness of $X_{\beta}$ and give necessary and sufficient conditions that $X_{\beta}$ be complete. Our approach is to regard $X_{\beta}$, under the mapping $x \rightarrow P_{x}$, as a subspace of $\operatorname{Hom}_{B}\left(B, X_{e}\right)$ with the strong operator topology (as in the latter part of the proof of Theorem 3.3).

THEOREM 3.5. In the strong operator topology $\operatorname{Hom}_{B}\left(B, X_{e}\right)$ is complete and contains $X_{e}$ as a dense subset. Consequently, $X_{\beta}$ is complete if, and only if, $X=$ $\operatorname{Hom}_{B}\left(B, X_{e}\right)$.

Proof. Suppose $\left\{P_{\alpha}\right\}$ is a net in $\operatorname{Hom}_{B}\left(B, X_{e}\right)$ that is Cauchy with respect to the strong operator topology. Then the limit $P(T)=\lim P_{\alpha}(T)$ exists in $X_{e}$ for each $T \in B$. Furthermore, if $S, T \in B$, then $P(S T)=\lim S P_{\alpha}(T)=S P(T)$ and by [12, Theorem 1], $P \in \operatorname{Hom}_{B}\left(B, X_{e}\right)$.

To see that $X_{e}$ is dense in $\operatorname{Hom}_{B}\left(B, X_{e}\right)$ let $P \in \operatorname{Hom}_{B}\left(B, X_{e}\right)$ and let $x_{\lambda}=P\left(E_{\lambda}\right)$. Then $P_{x_{\lambda}}(T)=P\left(T E_{\lambda}\right)$, so $P(T)=\lim P_{x_{\lambda}}(T)$ for each $T \in B$.

Since the strong operator topology on $\operatorname{Hom}_{B}\left(B, X_{e}\right)$ restricted to $X$ coincides with the $\beta$ topology on $X$ it follows that $X_{\beta}$ is complete if and only if $X=$ $\operatorname{Hom}_{B}\left(B, X_{e}\right)$.

THEOREM 3.6. If $X$ is a reflexive Banach space, then $X=\operatorname{Hom}_{B}\left(B, X_{e}\right)$.

Proof. For when $X$ is reflexive, $X=X_{e}$ and an appeal to [11, Corollary 8.10] completes the proof.

4. The dual of $X_{\beta}$. Let $X_{\beta}^{*}$ denote the linear space of all strictly continuous linear functionals on $X_{\beta}$. We denote by $X_{\beta}^{\prime}$ the space $X_{\beta}^{*}$ with the strong topology; that is, the topology of uniform convergence on the strictly bounded subsets of $X_{\beta}$. Hence $X_{\beta}^{\prime}$ denotes the dual space of $X_{\beta}$.

We now introduce a norm topology on $X_{\beta}^{*}$ which is weaker than the strong topology, namely the topology $\sigma^{*}$ of uniform convergence on the $\sigma$-bounded subsets of $X$. The topology $\sigma^{*}$ is induced by the norm $\left\|x^{\prime}\right\|=\sup \left\{\left|x^{\prime}(x)\right|:\|x\| \leqq 1\right\}$. We denote by $\left(X_{\beta}^{*}, \sigma^{*}\right)$ the linear space $X_{\beta}^{*}$ with the topology $\sigma^{*}$. 
Before proceeding we briefly review the work of Buck [1] on the space $X=C(S)$ as a Banach $C_{0}(S)$-module. Buck showed that $C(S)_{\beta}^{\prime}$ is the Banach space $M(S)$ of bounded regular Borel measures on $S$ which, as is well known, is the dual of $C_{0}(S)$. In particular, this meant that $C(S)_{\beta}^{\prime}=\left(C(S)_{\beta}^{*}, \sigma^{*}\right)$ and that $C(S)_{\beta}^{\prime}$ is a Banach space. The results we obtain in this section will readily imply these particular conclusions although it appears that $X_{\beta}^{\prime}$ is not in general a Banach space. However, a significant part of the proof that $C(S)_{\beta}^{\prime}=M(S)$ revolves around the fact that the strictly bounded sets in $C(S)_{\beta}$ are norm bounded. Under this condition on $X_{\beta}$ we will see that $X_{\beta}^{\prime}$ is the Banach space $\left(X_{\beta}^{*}, \sigma^{*}\right)$. With further notational development, we will also obtain a generalization of the fact that $C(S)_{\beta}^{\prime}=C_{0}(S)^{\prime}$, where the space $C_{0}(S)$ will be replaced in general by the Banach space $X_{e}$, while $C_{0}(S)^{\prime}$ will be replaced by $X_{e}^{c}$. This space will be defined shortly and it will be seen that $X_{e}^{c}=X_{e}^{\prime}$ when $X=C(S)$ and $B=C_{0}(S)$. Finally we will obtain a characterization of the equicontinuous subsets of $X_{\beta}^{*}$ which includes the work of Conway [4] on $C(S)_{\beta}^{*}$.

For notational convenience in developing the relationship between $X_{\beta}^{*}$ and $X_{e}$ we let $\phi\left(x^{\prime}\right)$ denote the restriction of an $x^{\prime} \in X_{\beta}^{*}$ to $X_{e}$. Note that $\phi$ defines a one-toone continuous linear mapping of $X_{\beta}^{\prime}$ into $X_{e}^{\prime}$.

Both $X$ and $X_{e}$ are left Banach $B$-modules. Consequently, $X^{\prime}$ and $X_{e}^{\prime}$ are right Banach $B$-modules under the operation $\left(x^{\prime}, T\right) \rightarrow x^{\prime} T$ and the usual norm, where $x^{\prime} T(x)=x^{\prime}(T x)$ for all $x \in X$, or respectively, all $x \in X_{e}$. Following the notation of Rieffel [11] we write $X_{e}^{c}=\left(X_{e}\right)^{c}=\left(X_{e}^{\prime}\right)_{e}$ and $X^{c}=\left(X^{\prime}\right)_{e}$ for the contragradient of these two spaces respectively and have

THEOREM 4.1. (1) As subsets of $X^{\prime}, X_{\beta}^{*}=X^{c}$ and $\phi$ is an isometric module isomorphism of $\left(X_{\beta}^{*}, \sigma^{*}\right)$ onto the Banach space $X_{e}^{c}$.

(2) The map $\phi$ is a continuous module isomorphism of $X_{\beta}^{\prime}$ onto $X_{e}^{c}$.

Proof. Any $x^{\prime} \in X^{c}$ is strictly continuous on $X$. Conversely, by Theorem 3.1, if $x^{\prime} \in X_{\beta}^{*}$ there is a $T \in B$ such that $\left|x^{\prime}(x)\right| \leqq\|T x\|$. Hence, $\left\|x^{\prime} E_{\lambda}-x^{\prime}\right\| \leqq\left\|T E_{\lambda}-T\right\|$ and by [11, Proposition 3.4], $x^{\prime} \in X^{c}$. A similar argument, along with the observation that $\phi$ is an isometry because $X_{e}$ is strictly dense in $X$, shows that the range of $\phi$ is $X_{e}^{c}$. The remaining conclusions are clear.

Corollary 4.2. $\left(X_{\beta}^{*}, \sigma^{*}\right)$ is a Banach space and consequently $X_{\beta}^{\prime}$ is complete.

Proof. For a proof, see [13, Proposition 3, p. 105].

THEOREM 4.3. The following statements are equivalent:

(1) $X_{\beta}^{\prime}$ is a Banach space.

(2) $X_{\beta}^{\prime}$ is a Fréchet space.

(3) $\phi^{-1}$ is a continuous mapping of $X_{e}^{c}$ onto $X_{\beta}^{\prime}$.

(4) $X_{\beta}^{\prime}$ is fully complete.

(5) $X_{\beta}^{\prime}=\left(X_{\beta}^{*}, \sigma^{*}\right)$ as topological vector spaces. 
Proof. If $X_{\beta}^{\prime}$ is a Banach space, then $X_{\beta}^{\prime}$ is a Fréchet space and consequently the mapping $\phi$ is open [13, p. 111 and p. 116] and $\phi^{-1}$ is continuous. But then by $\left[13\right.$, p. 119 and p. 111] $X_{\beta}^{\prime}$ is fully complete and [13, p. 116, Corollary 2] implies $X_{\beta}^{\prime}=\left(X_{\beta}^{*}, \sigma^{*}\right)$. Consequently $X_{\beta}^{\prime}$ is a Banach space completing the proof.

This yet leaves open the question of whether $X_{\beta}^{\prime}$ is always a Banach space. We can give an obviously sufficient condition for this.

COROLlary 4.4. If the $\sigma$ and $\beta$ bounded sets in $X$ are the same, then $X_{\beta}^{\prime}$ is a Banach space.

This leads us to the natural question of when are the $\sigma$ and $\beta$ bounded sets the same in $X$. An answer to this leads us back to another natural question: when are the topologies $\sigma$ and $\sigma^{\prime}$ the same? Before considering this we define yet another norm on the space $X$. Set $\|x\|_{1}=\sup \left\{\left|x^{\prime}(x)\right|: x^{\prime} \in X_{\beta}^{\prime},\left\|x^{\prime}\right\| \leqq 1\right\}$.

LEMMA 4.5. For any $x \in X,\|x\|^{\prime} \leqq\|x\|_{1} \leqq\|x\|$.

Proof. Clearly $\|x\|_{1} \leqq\|x\|$. Now let $\varepsilon>0$. Then there exists a $T \in B,\|T\| \leqq 1$, and a $y^{\prime} \in X_{e}^{\prime},\|y\| \leqq 1$, such that $\|x\|^{\prime} \leqq\left\|y^{\prime} T(x)\right\|+\varepsilon \leqq\|x\|_{1}+\varepsilon$. Hence $\|x\|^{\prime} \leqq\|x\|_{1}$, since $\varepsilon$ was chosen arbitrarily.

THEOREM 4.6. The following statements are equivalent:

(1) The strictly bounded sets are $\sigma$-bounded.

(2) The $\sigma^{\prime}$-bounded sets are $\sigma$-bounded.

(3) The strict closure of a $\sigma$-bounded set in $X_{e}$ is $\sigma$-bounded.

(4) The strict closure of a $\sigma$-bounded set in $X$ is $\sigma$-bounded.

(5) The norms \|\| and \|\|$_{1}$ are equivalent norms on $X$.

(6) The norms \|\| and \|\|$^{\prime}$ are equivalent norms on $X$.

Proof. By Lemma 4.5, (6) implies (5). Now we will show that (5) implies (4). Assume (5) holds and that $N$ is the strict closure of a $\sigma$-bounded subset of $X$. If we view $N$ as a family of continuous linear functionals on $\left(X_{\beta}^{*}, \sigma^{*}\right)$, then we have, by virtue of the uniform boundedness principle, that $N$ is bounded with respect to \|\|$_{1}$. Hence $N$ is $\sigma$-bounded, since $\|\quad\|$ and \|\|$_{1}$ are equivalent norms.

Clearly (4) implies (3) and we will show that (3) implies (2).

Let $K$ be a $\sigma^{\prime}$-bounded subset of $X$. Then $\bigcup_{\lambda} E_{\lambda}(K)$ is $\sigma$-bounded. Hence so is its strict closure, which evidently contains $K$.

To see that (2) implies (1), let $K$ be a strictly bounded set in $X$. Then consider $K$ as a subset of $\operatorname{Hom}_{B}\left(B, X_{e}\right)$ and apply the uniform boundedness principle.

To complete the proof, we will show that (1) implies (6). Let $W=\left\{x \in X:\|x\|^{\prime}\right.$ $\leqq 1\}$. Then $W$ is strictly bounded and by (1) is norm bounded. That is, there is a number $b$ such that $\|x\|^{\prime} \leqq 1$ implies $\|x\| \leqq b$. Hence $\|x\|^{\prime} \leqq\|x\| \leqq b\|x\|^{\prime}$.

Notice that statements (5) and (6) of Theorem 4.6 are respectively equivalent to the conditions that $\left(X,\|\|^{\prime}\right)$ be complete and $\left(X,\|\|_{1}\right)$ be complete. Furthermore, we have the following 
COROLlaRY 4.7. If $X_{\beta}$ is complete, then the strictly bounded sets in $X$ are norm bounded.

Proof. For if $X_{\beta}$ is complete, then by Theorem 3.5 the natural mapping $x \rightarrow P_{x}$ of $X$ into $\operatorname{Hom}_{B}\left(B, X_{e}\right)$ is in fact onto. Since $\left\|P_{x}\right\|=\|x\|^{\prime} \leqq\|x\|$ this mapping is continuous, and consequently the inverse mapping is continuous and the norms $\|\quad\|$ and $\|\quad\|^{\prime}$ on $X$ are equivalent. Hence $\left(X,\|\|^{\prime}\right)$ is complete, and by Theorem 4.6 the strictly bounded sets in $X$ are norm bounded.

It is apparent from a consideration of $B=X=C_{0}(S)$ that the converse of Corollary 4.7 does not hold.

We close this section with a characterization of the equicontinuous subsets of $X_{\beta}^{*}$ which yields the special results of Conway [4] for $C(S)_{\beta}$. Recall that a set $H \subset X_{\beta}^{*}$ is, by definition and Theorem 3.1, $\beta$-equicontinuous if and only if there is a $T \in B$ such that $\left|x^{\prime}(x)\right| \leqq\|T x\|$ for all $x^{\prime} \in H$. An equicontinuous set is necessarily bounded in $X_{\beta}^{\prime}$ by [13, Theorem 1, p. 67].

THEOREM 4.8. If $H$ is a subset of $X_{\beta}^{*}$, then the following statements are equivalent:

(1) $H$ is $\beta$-equicontinuous.

(2) $H$ is $\sigma^{*}$-bounded and $\left\|x^{\prime} E_{\lambda}-x^{\prime}\right\| \rightarrow 0$ uniformly on $H$.

(3) $H$ is $\sigma^{*}$-bounded and $\left\|\phi\left(x^{\prime}\right) E_{\lambda}-\phi\left(x^{\prime}\right)\right\| \rightarrow 0$ uniformly on $H$.

(4) There exists a $T \in B$ such that $H \subset\left\{y^{\prime} T: y^{\prime} \in X_{\beta}^{*},\left\|y^{\prime}\right\| \leqq 1\right\}$.

(5) There exists a $T \in B$ such that $\phi(H) \subset\left\{y^{\prime} T: y^{\prime} \in X_{e}^{c},\left\|y^{\prime}\right\| \leqq 1\right\}$.

Proof. If $H$ is $\beta$-equicontinuous, then by Theorem 3.1 there exists a $T \in B$ such that $\left|x^{\prime}(x)\right| \leqq\|T x\|$ for all $x^{\prime} \in H$. Consequently $H$ is $\sigma^{*}$-bounded by, $\|T\|$ and $\left\|x^{\prime} E_{\lambda}(x)-x^{\prime}(x)\right\| \leqq\left\|T E_{\lambda}-T\right\|\|x\|$. Hence (2) follows. It is clear that (2) implies (3) and that (3) implies (5) by virtue of Theorem 2.1 . It is equally apparent that (5) implies (4) and that (4) implies that $H$ is contained in the polar of some $\beta$-neighborhood in $X$, or equivalently, that $H$ is $\beta$-equicontinuous.

5. Examples and applications. In this section we apply our results to special cases of the strict topology considered by other authors as well as to some examples of our own origin.

We begin with a discussion of the strict topology induced by $B$ on the left Banach $B$-module $\operatorname{Hom}_{B}\left(B, X_{e}\right)$. This result includes the work of Buck [1] on $C(S)=\operatorname{Hom}_{C_{0}(S)}\left(C_{0}(S), C(S)_{e}\right)$ as well as that of Wang [18] on the space of multipliers of a commutative Banach algebra.

THEOREM 5.1. $\left[\operatorname{Hom}_{B}\left(B, X_{e}\right)\right]_{\beta}$ is a complete locally convex Hausdorff topological vector space in which the strictly bounded sets are bounded in the operator norm and whose strong dual is the Banach space $X_{e}^{c}$.

Proof. Let $W=\operatorname{Hom}_{B}\left(B, X_{e}\right)$. By [11, Theorem 4.5], $W_{e} \cong X_{e}$ under the mapping of an $x \in X_{e}$ to $T_{x} \in W_{e}$ defined by $T_{x}(S)=S x$. Then $\operatorname{Hom}_{B}\left(B, W_{e}\right)=\operatorname{Hom}_{B}\left(B, X_{e}\right)$ $=W$, so that by Theorem $3.5 W_{\beta}$ is complete. Hence by Corollary 4.7 the strictly 
bounded sets are norm bounded, and consequently by Theorems 4.1 and 4.4 we can regard $W_{\beta}^{\prime}$ as $W_{e}^{c} \cong X_{e}^{c}$.

As an application of Theorem 5.1 let $G$ be a locally compact Abelian group and consider $X=M(G)$ as a Banach $L^{1}(G)$-module under convolution. Then $X_{e}=L^{1}(G)$ and $X_{e}^{c}=L^{1}(G) * L^{\infty}(G)$. Hence, as is well known, $X_{e}^{c}$ is the space $C_{u}(G)$ of bounded uniformly continuous functions on $G$. Since $M(G)=\operatorname{Hom}_{B}\left(B, X_{e}\right)$, Theorem 5.1 applies and $M(G)_{\beta}^{\prime}=C_{u}(G)$.

For another example consider $L^{p}(G)$, for $1 \leqq p<\infty$, as a Banach $L^{1}(G)$-module under convolution. If $p=1$, then $L^{p}(G)=L^{1}(G)=M(G)_{e}$ and since $M(G)_{e}$ is strictly dense in $M(G)_{\beta}$ we obtain $L^{1}(G)_{\beta}^{\prime}=C_{u}(G)$. For $1<p<\infty, L^{p}(G)$ is reflexive and by Theorem 3.6 $L^{p}(G)=\operatorname{Hom}_{L^{1}(G)}\left(L^{1}(G), L^{p}(G)\right)$ and Theorem 5.1 applies. Since $X_{e}=X$ (see also [7]) we obtain the result that $L^{p}(G)_{\beta}^{\prime}=L^{q}(G)$ where $p+q=p q$.

Since the norm topology on $L^{p}(G)$ is strictly finer than $\beta$, by Theorem 3.2 , this example shows that even in the above fairly nice situation the strict topology on a space $X_{\beta}$ need not be the Mackey topology as was shown by Conway [4] for $C(S)_{\beta}$ with $S$ paracompact. We have been unable to obtain any general extension of this result.

We close with an application in a Hilbert space $X$ considered as a Banach $B$ module of the algebra $B$ of compact operators in $X$. In this setting, we obtain, by an appeal to Theorem 2.1, the result of Raimi [10] that the $k$ topology on $X$ (that is, the finest locally convex topology on $X$ which agrees with the weak topology on bounded sets) is in fact the strict topology.

\section{REFERENCES}

1. R. C. Buck, Bounded continuous functions on a locally compact space, Michigan Math. J. 5 (1958), 95-104.

2. P. Cohen, Factorization in group algebras, Duke Math. J. 26 (1959), 199-206.

3. H. S. Collins and W. H. Summers, Some applications of Hewitt's factorization theorem, Proc. Amer. Math. Soc. (to appear).

4. J. Conway, The strict topology and compactness in the space of measures, Trans. Amer. Math. Soc. 126 (1967), 474-486.

5. P. Curtis and A. Figá-Talamanca, "Factorization theorems for Banach algebras," in Function algebras, edited by F. J. Birtel, Scott, Foresman and Co., Chicago, Ill., 1966, pp. 169-185.

6. S. L. Gulik, T. S. Liu and A. C. M. van Rooij, Group algebra modules, Canad. J. Math. 19 (1967), 151-173.

7. E. Hewitt, The ranges of certain convolution operators, Math. Scand. 15 (1964), 147-155.

8. B. E. Johnson, Continuity of centralizers on Banach algebras, J. London Math. Soc. 41 (1966), 639-640.

9. C. Rickart, General theory of Banach algebras, Van Nostrand, Princeton, N. J., 1960.

10. R. Raimi, Compact transformations and the $k$-topology in Hilbert space, Proc. Amer. Math. Soc. 6 (1955), 643-646.

11. M. Rieffel, Induced Banach representations of Banach algebras and locally compact groups, J. Functional Analysis 1 (1967), 443-491. 
12. M. Rieffel, On the continuity of certain intertwining operators, centralizers, and positive linear functionals, Proc. Amer. Math. Soc. 20 (1969), 455-457.

13. A. P. Robertson and W. J. Robertson, Topological vector spaces, Cambridge Univ. Press, New York, 1964.

14. W. Rudin, Representations of functions by convolutions, J. Math. Mech. 7 (1958), 103-115.

15. D. Sentilles, Compact and weakly compact operators on $C(S)_{\beta}$, Illinios J. Math. (to appear).

16. D. Taylor, A characterization of Banach algebras with approximate unit, Bull. Amer. Math. Soc. 74 (1968), 761-766.

17. N. T. Varopoulos, Sur les formes positive d'une algèbre de Banach, C. R. Açad. Sci. Paris 258 (1964), 2465-2467.

18. Ju-kwei Wang, Multipliers of commutative Banach algebras, Pacific J. Math. 11 (1961), 1131-1149.

UNIVERSITY OF MISSOURI, Columbia, Missouri 